\title{
Increased risk of tuberculosis after gastrectomy and chemotherapy in gastric cancer: a 7-year cohort study
}

\author{
Shiang-Fen Huang • Chung-Pin Li • \\ Jia-Yih Feng $\cdot$ Yee Chao $\cdot$ Wei-Juin Su
}

Received: 27 December 2010/ Accepted: 18 February 2011/Published online: 19 April 2011

(c) The International Gastric Cancer Association and The Japanese Gastric Cancer Association 2011

\begin{abstract}
Background Gastrectomy for peptic ulcers and chemotherapy for malignancy are known risk factors for tuberculosis (TB). However, this relationship has rarely been investigated in patients with gastric cancer.

Methods In a retrospective cohort study from 2000 to 2006, data for 2215 patients diagnosed with gastric cancer
\end{abstract}

Electronic supplementary material The online version of this article (doi:10.1007/s10120-011-0037-1) contains supplementary material, which is available to authorized users.

\section{S.-F. Huang · J.-Y. Feng}

Department of Chest Medicine, Taipei Veterans General

Hospital, No. 201, Sec. 2, Shipai Rd., Beitou District, Taipei,

Taiwan, ROC

C.-P. $\mathrm{Li}$

Division of Gastroenterology, Department of Medicine,

Taipei Veterans General Hospital, Taipei, Taiwan, ROC

\section{J.-Y. Feng}

Institute of Clinical Medicine, School of Medicine,

National Yang-Ming University, Taipei, Taiwan, ROC

C.-P. Li · Y. Chao · W.-J. Su

School of Medicine, National Yang-Ming University,

Taipei, Taiwan, ROC

\section{Y. Chao $(\bowtie)$}

Chemoradiotherapy Division, Cancer Center, Taipei Veterans General Hospital, No. 201, Sec. 2, Shipai Rd., Beitou District, Taipei, Taiwan, ROC

e-mail: ychao@vghtpe.gov.tw

W.-J. Su ( $\square)$

Division of Pulmonary Immunology and Infectious Diseases, Department of Chest Medicine, Taipei Veterans General Hospital, No. 201, Sec. 2, Shipai Rd., Beitou District, Taipei, Taiwan, ROC

e-mail:wjsu@vghtpe.gov.tw at our hospital were compared with data from the Centers for Disease Control (CDC), Taiwan, to identify confirmed cases of TB.

Results In patients with gastric cancer without a history of gastrectomy and without previous anti-TB treatment, the overall crude incidence of new-onset TB was 788 per 100,000 person-years. Compared with the general population, the overall age-sex standardized incidence (SI) in gastric cancer patients was 134.3 (SI ratio [SIR]: 2.11, $p<0.05$ ), and the recurrence rate among patients with previous anti-TB treatment was $18 \%$ (4/22) after gastric cancer diagnosis. Gastrectomy was a significant risk factor for active TB incidence [SI 159 (95\% confidence interval [CI], 80-237, SIR: $2.5, p<0.05$ ), and chemotherapy alone seemed to be a risk factor, but was without statistical significance (SIR: 12.5, $p>0.05$ ). Multivariate analysis showed old age, male gender, previous anti-TB treatment, and gastrectomy as significant risk factors for TB. In stratified analysis, an interaction between old TB patterns on chest films and chemotherapy was observed.

Conclusions Old age, male gender, previous anti-TB treatment, and gastrectomy were significant risk factors for TB. An increased risk of TB incidence after chemotherapy was observed in patients with old TB pattern chest films initially, suggesting an interaction between chest film patterns and chemotherapy.

Keywords Chemotherapy - Gastric cancer . Gastrectomy - Standardized incidence ratio $\cdot$ Tuberculosis

\section{Introduction}

Tuberculosis (TB) is a widespread infectious disease, with an incidence of 139 cases per 100,000 person-years. 
In Taiwan, the annual incidence was 63.7 cases per 100,000 person-years in 2006. Risk factors related to the incidence of TB include older age, smoking, and male gender [1, 2]. A decrease in immunity has been hypothesized to be a risk factor for symptomatic TB and reactivation, especially in HIV-infected individuals [3], individuals with malignancies [4-10] including lung cancer and hematologic malignancies [11, 12], and individuals with malnutrition [1].

Some investigations have revealed a relationship between chemotherapy in malignancy and TB re-infection or reactivation [13]. However, the results included all malignancies, and other treatment methods such as gastrectomy or palliative treatment were not considered. Gastrectomy in patients with peptic ulcers is a risk for TB [14-16], and although gastrectomy and chemotherapy are standard treatments for gastric cancer, the role of these two treatments in the risk for TB has not been clearly demonstrated.

The aim of this study was to investigate the relationship between the incidence of TB and gastric cancer treatment. Information on this relationship may be able to provide clinical physicians with a better understanding of how to manage patients with gastric cancer with regard to TB.

\section{Patients, materials, and methods}

\section{Data source}

The patients were collected from the Cancer Center of Taipei Veterans General Hospital database, which contains records of newly diagnosed cancer patients since 1994, with a $96.9 \%$ follow-up rate.

\section{Study population}

All patients were retrospectively selected using the ICD-9 code 151 for gastric cancer from January 1, 2000 to December 31, 2006. Patients were excluded if they had a history of gastrectomy. Each patient had an individual profile including pathology, diagnosis information, TNM stage, comorbidity, treatment (gastrectomy, chemotherapy, radiation, radiofrequency, palliative treatment or without treatment), and follow-up status. Patients who received palliative surgery without gastrectomy and those who received radiotherapy alone were combined into the palliative treatment group. Each patient was followed until 2010 if still alive.

All patients were checked against the registration system of the Centers for Disease Control (CDC) in Taiwan or by chart review to identify a case of active TB. Details were obtained including notification date, sputum collection date, re-activation or first time diagnosis of TB, and confirmed status. Patients who had had anti-TB treatment before gastric cancer were also identified.

All information that allowed a specific individual patient to be identified was encrypted. This study was approved and informed consent was waived by the Institutional Review Board of Taipei Veterans General Hospital (VGHIRB No. 20102004IC).

\section{Incidence of TB}

Due to the possibility of negative sputum TB smears with positive TB cultures after $2-3$ months, only cases identified with TB two months after starting gastric cancer treatment were recognized as newly diagnosed active TB. Patients with $\mathrm{TB}$ notification within the two-month interval of gastric cancer diagnosis or treatment were categorized as concurrent TB with gastric cancer.

The baseline incidence $(n / N)$ of TB each year was calculated as the number of concurrent TB cases $(n)$ divided by the number of gastric cancer cases $(N)$ per year. The crude incidence (CI) of active TB was calculated as the number of new active TB cases per observation personyear under risk, and the crude incidence ratio (CIR) represented a subgroup comparison of incidence to the reference group. The age-sex standardized incidence (SI) was calculated by a direct method [17], setting the general population in Taiwan in 2006 as the reference population, and the standardized incidence ratio (SIR) was calculated by comparing the standardized active TB incidence with the incidence in the general population in 2006.

\section{Chest film review}

Chest films were reviewed by two board-certified pulmonologists, who were blinded to the patients' information. The images showed infiltration patterns and date of examination, and were categorized into subgroups as follows: (1) old healed TB (old TB pattern) if bronchovascular distortion, fibrotic bands, small calcified nodules, or bronchiectasis were observed as apical lesions in either upper lobe [18]; (2) metastasis pattern, which included a single nodule/mass or bilateral multiple nodules, with/ without mediastinum lymphadenopathy; (3) unilateral pleural effusion, with no other abnormality on the chest film; (4) mixed pattern, which represented both an old TB pattern and suspected metastasis pattern on the chest film; and (5) "others", which represented abnormal chest film images that could not be categorized into the other patterns. If there was no consensus between the two pulmonologists on the type of pattern, the image was discussed further until a consensus was reached. 


\section{Statistics}

Tests for significance were performed using the $\chi^{2}$ test and Fisher's exact test (2-sided) for categorical data, and Student's $t$-test for continuous data in univariate analysis. A logistic regression model was used for multivariate analysis. The data were analyzed using SPSS software version 17 (SPSS, Chicago, IL, USA), the incidence was calculated using Stata 10.0 (Stata Corporation, College Station, TX, USA), and the age-standardized incidence was calculated using StatsDirect software 2.7 (StatsDirect, Altrincham, Cheshire, UK).

\section{Results}

\section{Study population}

From January 1, 2000 to December 31, 2006, 2257 patients were selected. Forty-two of them with a history of subtotal or partial gastrectomy before the diagnosis of gastric cancer, and 6 patients with active TB within 6 months before the diagnosis of gastric cancer and receiving anti-TB treatment were excluded. In total, 2212 patients were enrolled, and they were divided into two groups: with and without previous anti-TB treatment.

Three of the 25 patients with previous anti-TB treatment and ten of the 2187 patients without a previous antiTB treatment history had concurrent $\mathrm{TB}$, and were excluded from the analysis. In total, 2177 patients without and 22 patients with previous anti-TB treatment were included in the analysis. The case selection is demonstrated in Fig. 1.

The characteristics of the 2199 patients with and without active TB are shown in Table 1. The majority of the patients were male $($ male/female ratio $=3.12)$, and they were older than the females $(p<0.05$, data not shown). The type of gastric cancer, clinical stage, veteran status, and presence of diabetes or other solid-organ malignancy were not related to the presence of active TB $(p>0.05)$. However, younger age, male gender, previous anti-TB treatment, and chest film findings during gastric cancer diagnosis were related to active TB $(p<0.05)$. The percentage of patients who received surgery alone was higher in patients with active TB (64.6 vs. $45.4 \%)$ and the percentages of patients who received chemotherapy alone or palliative treatment were lower in the active TB group (6.3 vs. $15.7 \%, 12.5$ vs. $19.8 \%$, respectively).

\section{Follow-up interval}

The mean follow-up interval ranged from zero to ten years. Seven hundred and twenty-six $(33.1 \%)$ of the patients were followed for more than three years, $717(32.6 \%)$ of the patients died, and $44(2 \%)$ were lost to follow-up within one year. The follow-up interval was related to the cancer cell type (pathology, $p<0.05$ ). There was no significant relationship between the interval of loss of follow-up and TNM stage, age, sex, treatment for gastric cancer, previous anti-TB treatment, and identification of active TB.
Fig. 1 Flow chart of case selection of patients with gastric cancer from 2000 to 2006 , Taipei Veterans General Hospital. Incidence $(A)$ : cumulative incidence of TB at the time of diagnosis of gastric cancer, calculated by the number of concurrent TB cases (n) divided by the number of gastric cancer cases $(N)$. Incidence $(\mathrm{A})=n / N \times$ 100,000. Incidence $(B)$ : overall incidence of active TB in patients without previous antiTB treatment, calculated by the number of identified active TB cases divided by observation person-years under risk

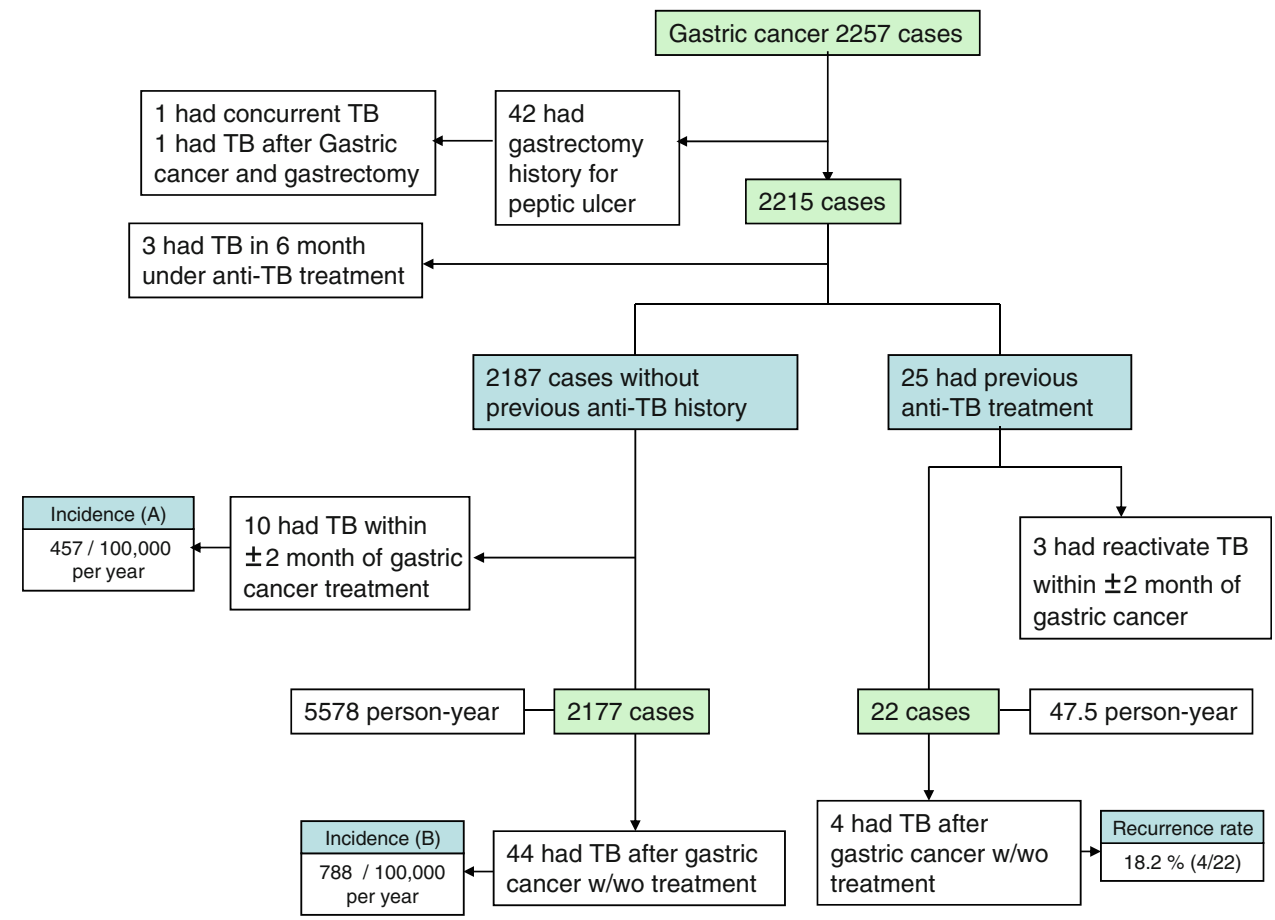


Table 1 Characteristics of the patients with active TB, with or without treatment, more than 2 months after gastric cancer was diagnosed from 2000 to $2006(n=2199)$

\begin{tabular}{|c|c|c|c|c|}
\hline Variable & $\begin{array}{l}\text { Total } \\
N(\%)\end{array}$ & $\begin{array}{l}\text { No active TB } \\
n=2151(\%)\end{array}$ & $\begin{array}{l}\text { Active TB } \\
n=48(\%)\end{array}$ & $p^{\mathrm{a}}$ \\
\hline Age at diagnosis (years) & & & & 0.022 \\
\hline$<40$ & $98(4.5)$ & $98(4.6)$ & $0(0)$ & \\
\hline $41-60$ & $431(19.6)$ & $428(19.9)$ & $3(6.3)$ & \\
\hline $61-80$ & $1333(60.7)$ & $1296(60.3)$ & $37(77.1)$ & \\
\hline$>80$ & $333(15.2)$ & $329(15.2)$ & $8(16.7)$ & \\
\hline Mean $\pm \mathrm{SE}$ & $68.5 \pm 0.29$ & $68.4 \pm 0.29$ & $74.1 \pm 1.1$ & $<0.001$ \\
\hline \multicolumn{5}{|l|}{ Gender } \\
\hline Male & $1671(75.9)$ & $1627(75.6)$ & $44(91.6)$ & 0.007 \\
\hline \multicolumn{5}{|l|}{ Previous TB complete treatment } \\
\hline Yes & $12(0.5)$ & $18(0.8)$ & $4(8)$ & 0.025 \\
\hline \multicolumn{5}{|l|}{ Veteran } \\
\hline Yes & $987(44.8)$ & $956(44.4)$ & $31(64.5)$ & 0.075 \\
\hline Cell type & & & & 0.24 \\
\hline Adenocarcinoma & $1728(78.6)$ & $1688(78.5)$ & $40(83.3)$ & \\
\hline Signet-ring cell carcinoma & $262(11.9)$ & $260(12.1)$ & $2(4.2)$ & \\
\hline Mucinous adenocarcinoma & $19(0.9)$ & $18(0.8)$ & $1(2.1)$ & \\
\hline GIST & $36(1.6)$ & $35(1.6)$ & $1(2.1)$ & \\
\hline Others & $154(7.0)$ & $150(7.0)$ & $4(8.3)$ & \\
\hline Cancer stage on diagnosis & & & & 0.12 \\
\hline Ia & $270(12.3)$ & $263(12.2)$ & $7(14.6)$ & \\
\hline $\mathrm{Ib}$ & $162(7.4)$ & $158(7.3)$ & $4(8.3)$ & \\
\hline II & $203(9.2)$ & $193(9.0)$ & $10(20.8)$ & \\
\hline IIIa & $235(10.7)$ & $231(10.7)$ & $4(8.3)$ & \\
\hline IIIb & $126(5.7)$ & $124(5.8)$ & $2(4.2)$ & \\
\hline IV & $814(37.0)$ & $803(37.3)$ & $11(22.9)$ & \\
\hline Missing data & $389(17.7)$ & $379(17.6)$ & $10(20.8)$ & \\
\hline Primary treatment for gastric cancer & & & & 0.1 \\
\hline Surgery without chemotherapy & $1007(45.8)$ & $976(45.4)$ & $31(64.6)$ & \\
\hline Surgery + chemotherapy & $407(18.5)$ & $399(18.5)$ & $8(16.7)$ & \\
\hline Chemotherapy alone & $339(15.5)$ & $336(15.67)$ & $3(6.3)$ & \\
\hline Local & $13(0.6)$ & $13(0.6$ & $0(0)$ & \\
\hline Palliative treatment & $433(19.7)$ & 427 (19.9) & $6(12.5)$ & \\
\hline \multicolumn{5}{|l|}{ Comorbidity } \\
\hline $\mathrm{DM}$ & $266(12.1)$ & 257 (11.9) & $9(18.8)$ & 0.093 \\
\hline Solid tumor & $169(7.7)$ & $164(7.6)$ & $5(10.4)$ & 0.489 \\
\hline Hematologic malignancy & $4(0.2)$ & $4(0.3)$ & $0(0)$ & \\
\hline CXR finding on diagnosis & & & & 0.099 \\
\hline Abnormal & & & & 0.003 \\
\hline Old TB pattern & $182(8.3)$ & $170(7.9)$ & $12(25.0)$ & \\
\hline Others & $98(4.5)$ & $96(4.5)$ & $2(4.2)$ & \\
\hline Mix & $85(3.9)$ & $85(4.0)$ & $0(0)$ & \\
\hline Metastatic & $165(7.5)$ & $164(7.6)$ & $1(2.1)$ & \\
\hline Unilateral pleural effusion & $47(2.1)$ & $46(1.2)$ & $1(2.1)$ & \\
\hline Normal & $1298(59.0)$ & $1276(59.3)$ & $22(45.8)$ & \\
\hline Not available & 324 (14.7) & 314 (14.6) & $10(20.8)$ & \\
\hline
\end{tabular}

GIST gastrointestinal stromal tumor, DM diabetes mellitus, $C X R$ chest $\mathrm{X}$-ray

${ }^{\text {a }} p$ value of 2 -sided $\chi^{2}$ test or Fisher-Freeman-Halton exact test for categorical data, or Student's $t$-test for continuous data (i.e., age) 
Incidence of $\mathrm{TB}$ and treatment method as risk factors for TB

As shown in Fig. 1, in patients with gastric cancer without a history of gastrectomy, the incidence of TB was 457 per 100,000 person-years at the time of diagnosis of gastric cancer. Of the 2177 patients without previous anti-TB treatment, the overall incidence was 788 per 100,000 person-years, and it was 8421 per 100,000 person-years among patients with previous anti-TB treatment, with a recurrence rate of $18.2 \%(4 / 22)$.

The age and sex-specific active TB incidence for the 2199 patients is demonstrated in Fig. 2, which shows that the incidence of active TB in gastric cancer patients was slightly higher than that in the general population. Figure $2 b$ shows the overall age-specific TB incidence according to treatment method. Both gastrectomy and chemotherapy had similar trends of a higher incidence than on diagnosis of gastric cancer or the general population.

The age-sex standardized incidence is shown in Table 2. For the 2199 gastric cancer patients, the overall standardized incidence of active TB was higher than that in the general population $(134.3 / 100,000$; 95\% confidence interval [CI] 80.9-187.6, SIR: 2.11, $p<0.05)$. Comparing the standardized TB incidence to that in the general population, patients who received palliative treatment had the lowest incidence (75.7/100,000; 95\% CI 16-135; SIR: $1.19, p>0.05)$, and the patients who received gastrectomy had a significantly higher incidence (159.1/100,000; 95\% CI 80.4-237; SIR: 2.5, $p<0.05)$. Patients receiving adjuvant chemotherapy or chemotherapy alone also had a trend of an increasing standardized incidence of active TB, although this trend was without statistical significance.

Data for patients without previous anti-TB treatment and the age-sex standardized incidence of active TB were recalculated (Table 3). In general, the overall standardized incidence of active TB in patients with gastric cancer was higher than that in the general population in Taiwan (SIR: 2.03, $p<0.05)$. Patients who received gastrectomy still had a significantly higher standardized incidence than the general population (SIR: 2.46, $p<0.05$ ).

Chest film pattern and interaction with treatment method for gastric cancer

When the chest films were categorized into old TB patterns, non-old TB patterns, and normal chest films, there was a difference in TB incidence between treatment methods (Table 4). Among patients with old TB patterns, treatment with chemotherapy alone had a trend of higher incidence of TB than treatment with surgery

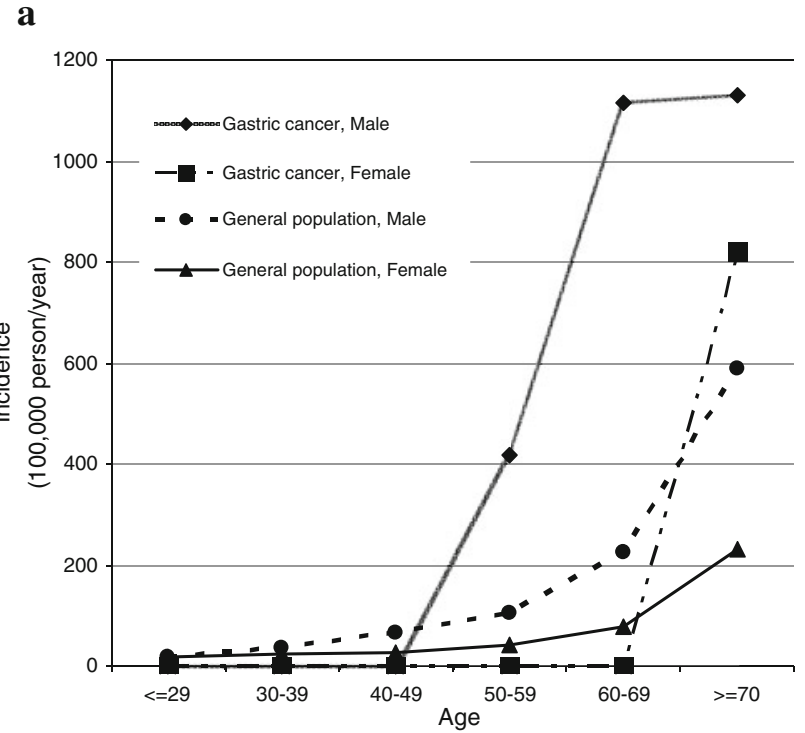

b

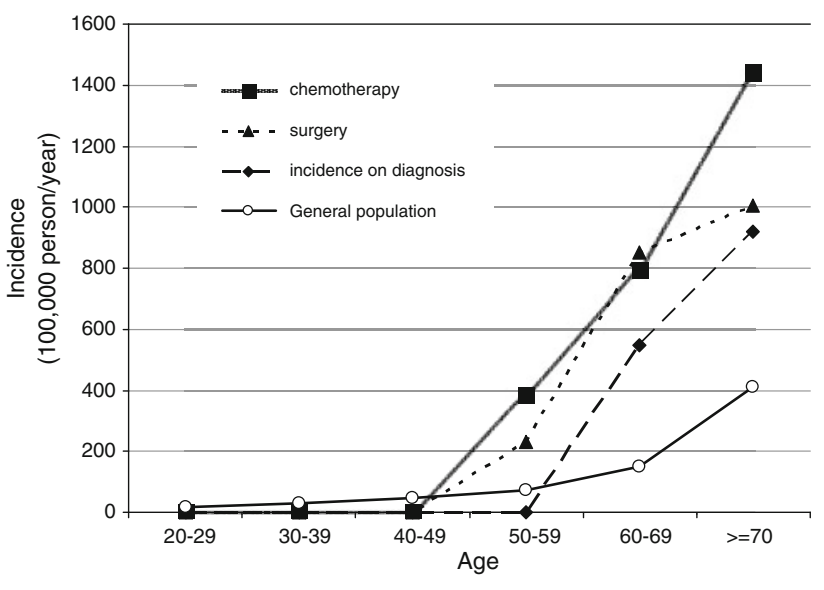

Fig. 2 Age- and sex-specific incidence of TB, compared to that in the general population in 2006 in Taiwan from the Centers for Disease Control (CDC) data. a Age- and sex-specific TB incidence. b Agespecific TB incidence by treatment for gastric cancer

alone (5681 vs. 2435, respectively, $\operatorname{CIR}=2.3,95 \%$ $\mathrm{CI}=0.3-17.3)$. This relationship was not present if the chest film showed other patterns $(\mathrm{CIR}=0.87,95 \% \mathrm{CI}$ 0.1-6.0). Furthermore, in patients who received palliative treatment, a slightly higher incidence of TB [crude incidence: 3514 vs. $2435, \mathrm{CIR}=1.4,95 \% \mathrm{CI}=0.2-11.2]$ but without statistics significance was found.

When the chest film images were categorized into normal, abnormal, and not available, there were no significant differences in the incidence of TB (data not shown).

Multivariate analysis

A multivariate logistic regression model for active TB was calculated (Table 5). After adjusting for the effects of age 
Table 2 Tuberculosis in patients after gastric cancer treatment $(n=2199)$

\begin{tabular}{|c|c|c|c|c|c|c|c|c|c|}
\hline $\begin{array}{l}\text { Treatment for } \\
\text { gastric cancer }\end{array}$ & $N$ & $\begin{array}{l}\text { Mean follow-up } \\
\text { (years) }\end{array}$ & Person-years & $n$ & $\begin{array}{l}\text { Crude } \\
\text { incidence }\end{array}$ & CIR & $p^{\mathrm{a}}$ & $\begin{array}{l}\text { Standard incidence } \\
(95 \% \mathrm{CI})^{\mathrm{b}}\end{array}$ & SIR \\
\hline Palliative treatment & 432 & 1.04 & 451.9 & 6 & 1327.7 & 1.6 & 0.27 & $75.7(16-135)$ & 1.19 \\
\hline Local treatment & 13 & 1.06 & 13.76 & 0 & 0 & & & 0 & \\
\hline Gastrectomy & 1007 & 3.985 & 3883.5 & 31 & 798.2 & 1 & - & $159.1(80.4-237)$ & $2.50^{*}$ \\
\hline Had chemotherapy & 744 & 1.74 & 1297.1 & 11 & 861.5.0 & 1.06 & 0.84 & $185.1(27.65-342)$ & 2.91 \\
\hline Gastrectomy $+\mathrm{CT}$ & 407 & 2.35 & 957.9 & 8 & 835.2 & 1.04 & 0.88 & $163.3(-6$ to 132$)$ & 2.56 \\
\hline CT alone & 339 & 0.96 & 323.99 & 3 & 940.9 & 1.15 & 0.75 & 793.9 ( -49 to 2836$)$ & 12.46 \\
\hline Total & 2192 & 2.56 & 5625.7 & 48 & 853.2 & & & $134.3(80.9-187.6)$ & $2.11 *$ \\
\hline
\end{tabular}

Incidence: cases per 100,000 person-years

$C T$ chemotherapy, $C I R$ crude incidence ratio, crude incidence compared to that in patients with gastric cancer after gastrectomy, SIR standardized incidence ratio, standardized incidence divided by incidence (63.7) in the general population, $C I$ confidence interval

${ }^{a} p$ value of CIR, 2-sided Mantel-Haenszel test, * $p$ value $<0.05$

b Age-sex standardized incidence, general population in Taiwan in 2006 as the reference group, by direct method. Use of Poisson model, improved approximate (Dobson) method for $95 \%$ confidence interval

Table 3 Tuberculosis in patients after gastric cancer treatment without known previous anti-TB treatment $(n=2177)$

\begin{tabular}{|c|c|c|c|c|c|c|c|c|c|}
\hline $\begin{array}{l}\text { Treatment for } \\
\text { gastric cancer }\end{array}$ & $N$ & $\begin{array}{l}\text { Mean follow-up } \\
\text { (years) }\end{array}$ & Person-years & $n$ & $\begin{array}{l}\text { Crude } \\
\text { incidence }\end{array}$ & CIR & $p^{\mathrm{a}}$ & $\begin{array}{l}\text { Standard incidence } \\
(95 \% \mathrm{CI})^{\mathrm{b}}\end{array}$ & SIR \\
\hline Palliative treatment & 418 & 1.05 & 438.5 & 3 & 684.2 & 0.56 & 0.35 & $39.8(8.2-116)$ & 0.62 \\
\hline Local treatment & 13 & 1.04 & 13.8 & 0 & 0.0 & - & - & 0 & \\
\hline Gastrectomy & 1001 & 3.84 & 3850.5 & 30 & 779.1 & 1 & - & $157(85-251)$ & $2.46^{*}$ \\
\hline Had chemotherapy & 744 & 1.72 & 1296.4 & 11 & 862.4 & 1.08 & 0.79 & $185.2(50-397)$ & 2.91 \\
\hline Gastrectomy $+\mathrm{CT}$ & 406 & 2.36 & 973.0 & 8 & 835.6 & 1.05 & 0.86 & $163.4(22-403)$ & 2.57 \\
\hline CT alone & 338 & 0.94 & 318.4 & 3 & 942.2 & 1.2 & 0.7 & $794(-49$ to 2836$)$ & 12.46 \\
\hline Total & 21876 & 2.6 & 5578.2 & 44 & 788.8 & & & $129.5(80-191)$ & $2.03 *$ \\
\hline
\end{tabular}

Incidence: cases per 100,000 person-years

$C I R$ crude incidence ratio, crude incidence compared to that in patients with gastric cancer after gastrectomy, SIR standardized incidence ratio, standardized incidence divided by incidence (63.7) in the general population, $C I$ confidence interval

${ }^{a} p$ value of CIR, 2-sided Mantel-Haenszel test, * $p$ value $<0.05$

b Age-sex standardized incidence, general population in Taiwan in 2006 as the reference group, by direct method. Use of Poisson model, improved approximate (Dobson) method for $95 \%$ confidence interval

and sex, both previous anti-TB treatment and gastrectomy were significant factors $(p<0.05)$ regarding active TB. There was no increased risk when considering the gastric cancer stage $(p>0.5)$; however, both gastrectomy $(p=0.03$, relative risk $[\mathrm{RR}]=3.3,95 \%$ CI 1.13-9.7) and old TB pattern on chest film were still significant risk factors $(p<0.001, \mathrm{RR}=3.6,95 \% \mathrm{CI}$ 1.76-7.4, data not shown). Compared to the patients who received palliative treatment, patients who received adjuvant chemotherapy showed a borderline relationship to active TB $(p=0.058)$. Subgroup analysis for patients without previous anti-TB treatment was also performed. The results showed that the chest film pattern was still related to the presence of TB (Supplement 1), and gastrectomy and adjuvant chemotherapy remained significant factors for active TB.

\section{Discussion}

The present study was a retrospective cohort study of the incidence of active TB among patients with gastric cancer. Of particular note is that this study introduced the method of age-sex standardized incidence compared to that in the general population, and analysis of the interaction of chest film findings and treatment was performed.

According to a previous survey of the health insurance database in Taiwan, the crude incidence of TB among patients with gastric cancer was 324 per 100,000 personyears [19]. Our baseline incidence was 457 per 100,000 person-years and the follow-up incidence of newly active TB after gastric cancer diagnosis was 788 per 100,000 person-years overall among patients without previous antiTB treatment (Table 3). There are several reasons for the 
Table 4 Interaction between chest plain film images on diagnosis and treatment method for gastric cancer $(n=2199)$

\begin{tabular}{|c|c|c|c|c|c|c|c|c|}
\hline CXR finding & $N$ & Person-years & $n$ & Incidence & CIR & $(95 \% \mathrm{CI})$ & $\begin{array}{l}\text { Standard incidence } \\
(95 \% \mathrm{CI})\end{array}$ & SIR \\
\hline \multicolumn{9}{|l|}{ Non-old TB pattern on CXR } \\
\hline Gastrectomy & 811 & 3183.6 & 17 & 534.0 & 1 & - & $122.8(48.5-230)$ & 1.93 \\
\hline Have chemotherapy & & & & 616.9 & 1.15 & $(0.5-2.9)$ & $157.1(18-415)$ & 2.47 \\
\hline Gastrectomy + chemotherapy & 321 & 740.4 & 5 & 675.3 & 1.26 & $(0.5-3.4)$ & $137.9(3.3-403)$ & 2.16 \\
\hline Chemotherapy & 259 & 232.2 & 1 & 430.6 & 0.87 & $(0.1-6.0)$ & $813.1(20.6-4530)$ & 12.76 \\
\hline Palliative treatment & 293 & 243.5 & 3 & 1232.3 & 2.3 & $(0.7-7.6)$ & $89.8(18.5-262)$ & 1.41 \\
\hline \multicolumn{9}{|l|}{ Old TB on CXR } \\
\hline Gastrectomy & 100 & 369.5 & 9 & 2435.8 & 4.56 & $(2.1-9.5)^{*}$ & $192.7(77-397)$ & $3.03 *$ \\
\hline Have chemotherapy & & & & 3037.5 & 6.7 & $(1.9-23.8)^{*}$ & $488.8(59-1765)$ & 7.67 \\
\hline Gastrectomy + chemotherapy & 19 & 48.2 & 1 & 2072.9 & 3.88 & $(0.6-25.2)$ & $861.9(21-4802)$ & 13.53 \\
\hline Chemotherapy & 26 & 17.6 & 1 & 5681.8 & 10.6 & $(2.1-53)^{*}$ & $799.4(20-4454)$ & 12.55 \\
\hline Palliative treatment & 36 & 28.5 & 1 & 3514.4 & 6.5 & $(1.1-37.7)^{*}$ & $902.2(22-5026)$ & 14.16 \\
\hline
\end{tabular}

$p$ value: univariate analysis, obtained by the 2-tailed Mantel-Haenszel test

$C I R$ crude incidence ratio, $C I$ confidence interval, SIR standardized incidence ratio, standardized incidence divided by incidence (63.7) in the general population

$* p$ value $<0.05$

Table 5 Multivariate logistic regression model for identification of active TB after gastric cancer treatment $(n=2199)$

\begin{tabular}{|c|c|c|c|c|c|}
\hline \multirow[t]{2}{*}{ Variable } & \multirow[t]{2}{*}{$N$} & \multirow[t]{2}{*}{ aRR } & \multicolumn{2}{|c|}{$95 \%$ CI } & \multirow[t]{2}{*}{$p$} \\
\hline & & & Lower & Upper & \\
\hline \multicolumn{6}{|l|}{ Sex } \\
\hline Male & 1671 & 2.32 & 0.81 & 6.6 & 0.117 \\
\hline Female & 528 & - & - & - & - \\
\hline Age & 2199 & 1.03 & 1.01 & 1.06 & $0.041 *$ \\
\hline \multicolumn{6}{|l|}{ History of anti-TB treatment } \\
\hline No & 2177 & - & - & - & - \\
\hline Yes & 22 & 8.1 & 2.2 & 29.6 & $0.002 *$ \\
\hline \multicolumn{6}{|l|}{ CXR finding } \\
\hline Old TB pattern & 182 & 3.15 & 1.5 & 6.6 & $0.002 *$ \\
\hline Non old TB pattern & 1693 & - & - & - & - \\
\hline Not available & 324 & 2.5 & 1.17 & 5.35 & 0.18 \\
\hline Treatment for gastric cancer & & & & & $0.038 *$ \\
\hline Gastrectomy alone & 1007 & 3.76 & 1.43 & 9.9 & $0.007 *$ \\
\hline Local & 13 & 0 & 0 & & 0.99 \\
\hline CT alone & 339 & 1.06 & 0.25 & 4.5 & 0.93 \\
\hline Gastrectomy + CT & 407 & 3.12 & 0.96 & 10.0 & 0.058 \\
\hline Palliative surgery or no treatment & 433 & - & - & - & - \\
\hline DM & 266 & 1.5 & 0.79 & 3.2 & 0.28 \\
\hline Malignancy & 173 & 1.04 & 0.38 & 2.8 & 0.94 \\
\hline
\end{tabular}

$a R R$ adjusted risk ratio estimated by the logistic regression model, $C I$ confidence interval, $C T$ chemotherapy, $D M$ diabetes mellitus

$* p$ value $<0.05$

high incidence: (1) the source of our database was patients older than the general population; more than $75 \%$ of the patients were older than 60 years of age, and older age is a risk factor for TB [20]. (2) There were more males (75.6\%) in this study, and male gender is also related to a high incidence of TB. (3) Our population was limited to patients with gastric cancer, leading to a variable and higher range of incidence.

Our results showed that the patients who underwent gastrectomy had a significantly higher incidence of TB than the general population [SIR 2.5 (95\% CI 1.26-3.72)], and chemotherapy alone had a similar trend [SIR 12.46 (95\% CI -0.76 to 44.5$)]$. When patients with previous antiTB treatment were excluded, the relationship between gastrectomy and TB was unchanged [SIR 2.46 (95\% CI 1.33-3.94)]. This suggests that gastrectomy is an independent risk factor for TB that might not be affected by the history of previous anti-TB treatment. This result is compatible with previous studies in which gastrectomy [14] and chemotherapy for malignancy had an increased risk of TB [21]. However, the small sample size in our study limits the statistical inference, and further studies with large populations should be considered.

Some studies have shown conflicting conclusions about short-term intensive cyclic chemotherapy for hematological malignancy not being related to TB reactivation [22]. Previous studies have included all TB patients with nonspecific solid-organ malignancy, or only cohorts with hematologic malignancy with TB and concurrent chemotherapy. The present study focused on gastric cancer patients and was limited to new-onset (or recurrent) TB diagnosed two months after gastric cancer treatment, rather than concurrent $\mathrm{TB}$, which is more specific in patients with gastric cancer.

An interaction between old TB patterns on chest films and chemotherapy was found in our study. This result is also compatible with previous research. One case-controlled study showed that chronic renal failure, old healed 
TB on chest X-ray, and anti-cancer chemotherapy were risk factors for TB in patients with malignancy [21]. That study also found an interaction between old healed TB on chest X-ray and chemotherapy for malignancy. However, in our study, the age-standard incidence ratio revealed conflicting findings (i.e., no difference in incidence ratio [SIR] between patients who received chemotherapy with or without old TB patterns on chest film). This could be explained by the limited case numbers and because the standardized incidence was diluted by the normal distribution in the general population. When the patients who received chemotherapy (adjuvant chemotherapy or chemotherapy alone) were combined, the bias appeared to be diminished and the age-standardized incidences $(95 \% \mathrm{CI})$ were $157.1(18-415)$ and 488.8 (59-1765), and SIRs were 2.47 and 7.47, respectively. Among patients without a previous history of anti-TB treatment who had old TB chest X-ray patterns, the interaction between TB and chemotherapy remained (Supplement 2). This may indicate that asymptomatic or latent $\mathrm{TB}$ is a risk for $\mathrm{TB}$ incidence after chemotherapy for gastric cancer. However, a lack of evidence for latent status is a limitation of the present study. Further immunological tests, including interferon gamma release assay (IGRA) tests [23-27] and follow-up should be conducted, although the role of interferon-gamma in high-burden areas remains controversial [28].

In the present survey, $14.5 \%$ of the patients did not have available chest film images, and 8 of them had TB after gastric cancer treatment. Chest films were not routinely checked during the early years of the hospital, and $70 \%$ (227/324) of them were diagnosed before the end of 2002. Because an old TB pattern on chest film was a significant risk factor for TB and risk of incidence after chemotherapy, routine chest X-ray check-ups are necessary and further prospective studies should be performed.

There were some limitations to the present study with regard to the risk of chest film pattern and TB incidence. First, not all patients were routinely checked for sputum acid-fast stains, due to either a lack of symptoms or signs or because of the invasive nature of the diagnostic procedure (i.e., bronchoalveolar lavage). This lack of routine checking may have caused bias in the incidence of active TB. In addition, in patients with old healed TB patterns, sputum check-ups might have been performed more aggressively than in those without old healed TB patterns, which may have led to an earlier detection of active $\mathrm{TB}$ and a lead time bias and a higher incidence (lower observation person-years under risk) than that in patients without old healed TB patterns on chest $\mathrm{X}$-ray.

The stage of the disease was a confounding factor with choice of treatment. In our study, multivariate analysis showed no increased risk among different cancer stages $(p \geq 0.5)$. Sub-population analysis according to clinical TNM stage should be done to adjust for the confounding effect; however, this analysis was not performed due to the limited sample size in our study. Further investigations such as multicenter studies might solve this problem.

Some patients with poor clinical status may have received palliative treatment. The incidence of active TB among these patients is indicated in Tables 2 and 3; there was no difference between the group with palliative treatment and general population $(p>0.05)$. This finding indicates that the neoplasm itself may be not associated with active TB itself. The lack of a European Cooperative Oncology Group (ECOG) score and information on nutritional status in our database were limitations, and such information should be included in future investigations.

A decrease in immunity has been hypothesized to be a risk factor for symptomatic $\mathrm{TB}$ and reactivation, by reducing the expression of gamma interferon, tumor necrosis factor alpha, and other mycobactericidal substances which may selectively compromise portions of the cell-mediated response that are important for containing and restricting TB [29, 30]. Gastrectomy can cause a poor nutritional status leading to poor immunity, and TB also leads to a poor nutritional status [31]. Micronutrients, including vitamins A, C, E, B, and folic acid, and minerals zinc, copper, selenium, and iron have key roles in metabolic pathways, cellular function, and immune competence [32]. The concentrations of these entities may play a role in the host defense against TB [33]. There have been few studies on the influence on TB exerted by specific chemotherapy agents such as 5-fluorouracil (5-FU)/platinumbased regimens for gastric cancer [34]. Because 5-FU is an antagonist of folic acid metabolism and because platinum may influence the cell cycle, both types of agents might play a role in immunity to $\mathrm{TB}$, and the mechanism needs to be investigated further.

Some risk factors were not available for examination in this retrospective study, such as advanced HIV infection, chronic renal failure, infliximab therapy, poorly controlled diabetes, underweight $(<10 \%$ below normal), malnutrition, use of glucocorticoids, general condition (i.e., ECOG score), and silicosis. Due to the limited sample size, detailed gastrectomy methods (subtotal, total, partial gastrectomy) showed no influence on TB (data not shown), and subgroup analysis of different chemotherapy agents was also not performed. In order to differentiate the risks for $\mathrm{TB}$ reactivation after chemotherapy, more gastric cancer patients should be enrolled in future multicenter investigations. Newly developed screening methods such as IGRAs should also be conducted to verify the status of latent $\mathrm{TB}$ and reactivation after gastric cancer treatment. 


\section{Conclusions}

In summary, this study demonstrated a higher incidence of TB infection after gastrectomy than that in the general population. Surgery might be an independent risk factor for the incidence of TB. Patients with previous anti-TB treatment also had a higher risk of reactivation after chemotherapy. Among patients with gastric cancer at the time of the diagnosis of TB, a chest X-ray is necessary to identify old healed TB patterns, as these patterns might increase the risk of $\mathrm{TB}$ incidence during the management of gastric cancer.

Acknowledgments We are grateful for the opinions and recommendations of the editors and reviewers. Some of the article content will be published at the 42nd World Conference on Lung Health (IUATLD) in October 2011.

\section{References}

1. Nava-Aguilera E, Andersson N, Harris E, Mitchell S, Hamel C, Shea B, et al. Risk factors associated with recent transmission of tuberculosis: systematic review and meta-analysis. Int J Tuberc Lung Dis. 2009;13(1):17-26.

2. Zevallos M, Justman JE. Tuberculosis in the elderly. Clin Geriatr Med. 2003;19(1):121-38.

3. Corbett EL, Churchyard GJ, Clayton TC, Williams BG, Mulder D, Hayes RJ, et al. HIV infection and silicosis: the impact of two potent risk factors on the incidence of mycobacterial disease in South African miners. AIDS. 2000;14(17):2759-68.

4. Jackson A, Garber PE, Post GW. Coexistent pulmonary tuberculosis and malignancy. Dis Chest. 1957;32(2):189-97.

5. Kaplan MH, Armstrong D, Rosen P. Tuberculosis complicating neoplastic disease. A review of 201 cases. Cancer. 1974;33(3):850-8.

6. Feld R, Bodey GP, Groschel D. Mycobacteriosis in patients with malignant disease. Arch Intern Med. 1976;136(1):67-70.

7. Patel KC, Shah DP, Sheth SM, Kamat SR. Association of tuberculosis with malignancy. J Postgrad Med. 1977;23(4):193-6.

8. Ibrahim EM, Uwaydah A, al-Mulhim FA, Ibrahim AM, el-Hassan AY. Tuberculosis in patients with malignant disease. Indian $\mathrm{J}$ Cancer. 1989;26(2):53-7.

9. Libshitz HI, Pannu HK, Elting LS, Cooksley CD. Tuberculosis in cancer patients: an update. J Thorac Imaging. 1997;12(1):41-6.

10. De La Rosa GR, Jacobson KL, Rolston KV, Raad, II, Kontoyiannis DP, Safdar A. Mycobacterium tuberculosis at a comprehensive cancer centre: active disease in patients with underlying malignancy during 1990-2000. Clin Microbiol Infect. 2004;10(8):749-52.

11. Stefan DC, Kruis AL, Schaaf HS, Wessels G. Tuberculosis in oncology patients. Ann Trop Paediatr. 2008;28(2):111-6.

12. Khan B, Ahmed P, Ullah K, Hussain CA, Hussain I, Raza S. Frequency of tuberculosis in haematological malignancies and stem cell transplant recipients. J Coll Physicians Surg Pak. 2005;15(1):30-3.

13. Kim DK, Lee SW, Yoo CG, Kim YW, Han SK, Shim YS, et al. Clinical characteristics and treatment responses of tuberculosis in patients with malignancy receiving anticancer chemotherapy. Chest. 2005;128(4):2218-22.

14. Yokoyama T, Sato R, Rikimaru T, Hirai R, Aizawa H. Tuberculosis associated with gastrectomy. J Infect Chemother. 2004;10(5):299-302.
15. Boman K. Tuberculosis occurring after gastrectomy. Acta Chir Scand. 1956;110(6):451-7.

16. Thorn PA, Brookes VS, Waterhouse JA. Peptic ulcer, partial gastrectomy, and pulmonary tuberculosis. Br Med J. 1956;1(4967): 603-8.

17. National Center for Health Statistics. Report of the workshop on age adjustment. Vital and Health Statistics. 1998;Series 4. No. 30.

18. Im JG, Itoh H, Shim YS, Lee JH, Ahn J. Pulmonary tuberculosis: CT findings - early active disease and sequential change with antituberculous therapy. Radiology. 1993;186:653-60.

19. Wu CY, Hu HY, Pu CY, Huang N, Shen HC, Li CP, Chou YJ. Aerodigestive tract, lung and haematological cancers are risk factors for tuberculosis: an 8-year population-based study. Int $\mathbf{J}$ Tuberc Lung Dis. 2011;15(1):1-6.

20. Tan KK, Cherian A, Teo SK. Tuberculosis in the elderly. Singapore Med J. 1991;32(6):423-6.

21. Kim HR, Hwang SS, Ro YK, Jeon CH, Ha DY, Park SJ, et al. Solid-organ malignancy as a risk factor for tuberculosis. Respirology. 2008;13(3):413-9.

22. Nair R, Prabhash K, Sengar M, Bakshi A, Gujral S, Gupta S, et al. The effect of short-term intensive chemotherapy on reactivation of tuberculosis. Ann Oncol. 2007;18(7):1243-5.

23. Latorre I, De Souza-Galvão M, Ruiz-Manzano J, Lacoma A, Prat C, Fuenzalida L, Altet N, Ausina V, Domínguez J. Quantitative evaluation of T-cell response after specific antigen stimulation in active and latent tuberculosis infection in adults and children. Diagn Microbiol Infect Dis. 2009;65:236-46.

24. Chee CB, Barkham TM, Khinmar KW, Gan SH, Wang YT. Quantitative T-cell interferon-gamma responses to Mycobacterium tuberculosis-specific antigens in active and latent tuberculosis. Eur J Clin Microbiol Infect Dis. 2009;28:667-70.

25. Janssens JP, Roux-Lombard P, Perneger T, Metzger M, Vivien R, Rochat T. Quantitative scoring of an interferon-gamma assay for differentiating active from latent tuberculosis. Eur Respir J. 2007;30:722-8.

26. Arend SM, Thijsen SF, Leyten EM, Bouwman JJ, Franken WP, Koster BF, Cobelens FG, van Houte AJ, Bossink AW. Comparison of two interferon-gamma assays and tuberculin skin test for tracing tuberculosis contacts. Am J Respir Crit Care Med. 2007;175:618-27.

27. Goletti D, Vincenti D, Carrara S, Butera O, Bizzoni F, Bernardini G, Amicosante M, Girardi E. Selected RD1 peptides for active tuberculosis diagnosis: comparison of a gamma interferon wholeblood enzyme-linked immunosorbent assay and an enzymelinked immunospot assay. Clin Diagn Lab Immunol. 2005;12:1311-6.

28. Davidow AL. Interferon-gamma release assay test characteristics depend upon the prevalence of active tuberculosis. Int $\mathbf{J}$ Tuberc Lung Dis. 2009;13:1411-5.

29. Chandra RK. Nutrition and immunity: lessons from the past and new insights into the future. Am J Clin Nutr. 1991;53(5): 1087-101.

30. Perronne C. Tuberculosis, HIV infection, and malnutrition: an infernal trio in central Africa. Nutrition. 1999;15(4):321-2.

31. Babcock A, editor. Nutrition and tuberculosis: a review of the literature and considerations for TB control programmes. Washington: US Agency for International Development; 2008.

32. Chandra RK. Nutritional deficiency and susceptibility to infection. Bull World Health Organ. 1979;57(2):167-77.

33. Karyadi E, Schultink W, Nelwan RH, Gross R, Amin Z, Dolmans $\mathrm{WM}$, et al. Poor micronutrient status of active pulmonary tuberculosis patients in Indonesia. J Nutr. 2000;130(12):2953-58.

34. Tsukamura M. In vitro susceptibility of mycobacteria, especially of Mycobacterium intracellulare, to 5-fluorouracil and pattern of development of resistance of Mycobacterium tuberculosis to the drug. Microbiol Immunol. 1979;23(5):427-9. 